\title{
Feasibility and long-term efficacy of hysteroscopic myomectomy for myomas with intramural development by the use of non-electrical "cold" loops
}

\author{
Francesco Paolo Giuseppe Leone - Stefania Calabrese • \\ Carmelo Marciante • Irene Cetin • Enrico Ferrazzi
}

Received: 7 August 2011 /Accepted: 4 October 2011 /Published online: 26 October 2011

(C) Springer-Verlag 2011

\begin{abstract}
This study aimed to assess the feasibility and the short- and long-term efficacy of hysteroscopic myomectomy for submucous myomas with intramural development [grade 1 (G1) and grade 2 (G2)] by using non-electrical "cold" loops and to verify the role of preoperative variables on surgical outcomes. Symptomatic (bleeding and infertility) premenopausal patients with the diagnosis of submucous myoma at transvaginal sonography, and with G1 and G2 grading at sonohysterography, were included in this prospective study. Hysteroscopic myomectomy was performed by the combined monopolar electrical slicing and traction-andleverage manoeuvres by non-electrical "cold" loops. One hundred fifty-nine patients were recruited, and 169 procedures were performed. Operating time, fluid deficit, complications and complete resection at first procedure (perioperative outcomes) and persisting symptoms and additional surgery for persisting symptoms (long-term outcomes) were not significantly different for G1 vs. G2 myomas. Perioperative outcomes were significantly different when comparing myoma mean diameter $<3.0$ vs. $\geq 3.0 \mathrm{~cm}$ ( 75 th percentile). Multivariate analysis and multiple regression analysis proved that myoma mean diameter was the only significant variable for surgical outcomes. Hysteroscopic myomectomy by non-
\end{abstract}

Electronic supplementary material The online version of this article (doi:10.1007/s10397-011-0706-4) contains supplementary material, which is available to authorized users.

F. P. G. Leone $(\varangle) \cdot$ S. Calabrese $\cdot$ C. Marciante $\cdot$ I. Cetin Department of Obstetrics and Gynecology,

Clinical Sciences Institute L. Sacco, University of Milan,

Via G.B. Grassi 74,

20157 Milan, Italy

e-mail: leone.francesco@hsacco.it

E. Ferrazzi

Childrens' Hospital Buzzi, University of Milan,

Milan, Italy electrical "cold" loops is an effective and efficient treatment for submucous myomas with intramural development. Myoma mean diameter is the only significant preoperative variable for perioperative outcome, while myoma grading loses its role as a prognostic factor.

Keywords Hysteroscopic myomectomy · Non-electrical "cold" loops · Submucous myoma · Long-term follow-up · Hysteroscopic complications

\section{Background}

Hysteroscopic myomectomy is an efficient and effective technique and is considered the standard of care for conservative surgical removal of symptomatic (bleeding and infertility) submucous myomas [1-3]. The size and the different grading $(\mathrm{G})$ of intramural extension of submucous myomas determine the chance of a complete resection and are strictly related to the risk of intraoperative complications (haemorrhage, perforation and fluid overload) [4-8].

Resectoscopic myomectomy is usually performed by the slicing technique with electrical loops. This technique is optimal for intracavitary myomas (G0) but may lead to partial section of the myometrial fibres on the uterine wall when myomas have a partial (G1) or prevalent (G2) intramural development. Moreover, thermal injury by mono- or bipolar unmodulated pure cutting electrosurgery might extend to more than $1.0 \mathrm{~mm}[9,10]$, with additional damage to the myometrium and to the endometrial cavity. This might lead to late fertility complications, such as intrauterine synechiae [11].

In mid-1990s, non-electrical loops ("cold loops") were introduced as an additional tool for hysteroscopic myomectomy [12]. The use of cold loops to enucleate the intramural 
portion of submucous myomas might avoid the unnecessary extensive electrical cut of surrounding myometrial fibres and the thermal injury of the adiacent myometrium. This "mechanical" approach minimises direct myometrial damage and unnecessary fibrosis in the area of surgical intervention. Recently, this pioneering technique has proved to be effective in a small series of large submucous myomas ( $\geq 5 \mathrm{~cm}$ ) [13]; however, it has never been scrutinized in large and long-term prospective studies. The aim of this study was to assess the feasibility and the short- and long-term efficacy of the combined use of non-electrical "cold" loops and monopolar electrical loops for resectoscopic removal of G1 and G2 submucous myomas in a prospective series of symptomatic (bleeding and infertility) premenopausal patients.

\section{Methods}

Patients Premenopausal patients complaining abnormal uterine bleeding and/or infertility with G1 and G2 myomas were consecutively recruited between January 2000 and December 2009. Relevant demographic data were recorded. The study design was approved by the institutional review board.

Sonographic assessment Number, site and size of myomas, the myometrial free margin and endometrial thickness and pattern were assessed by high-quality transvaginal sonography and saline contrast sonohysterography (SCSH). SCSH was always performed according to previously published 2D and 3D imaging criteria in order to preoperatively classify myoma grading $[14,15]$. Grade 1 (G1) was defined as a sessile fibroid, with the endocavitary part protruding into the cavity dilated by saline solution $\geq 50 \%$, and grade 2 (G2) with the endocavitary part $<50 \%$ and prevalent intramural development. The myometrial free margin was considered the shortest distance between the outer margin of the myoma and the perimetrium as assessed by SCSH. Inclusion criteria were: number $\leq 3$ and size $\leq 5 \mathrm{~cm}$ of submucous myomas and myometrial free margin $\geq 3 \mathrm{~mm}$. The mean diameter of myomas was calculated from the three diameters: $(d 1+d 2+d 3) / 3$.

Medical preoperative treatment $\mathrm{GnRH}$ analogue triptorelin or leuprorelin were prescribed for 2-3 months to patients with myomas $>3 \mathrm{~cm}$, in order to reduce myoma volume, to induce endometrial hypotrophy, and/or for patients suffering from secondary anaemia.

Hysteroscopic removal When no preoperative medication was prescribed, all patients underwent surgery in the early proliferative phase of the cycle. Resectoscopic procedures were performed using a rigid $8-\mathrm{mm}(27 \mathrm{Fr})$ continous-flow resectoscope for intrauterine high-frequency electrosurgery with rotatable inner tube and a $12^{\circ}$ fore-oblique telescope (Karl Storz GmbH \& Co., Germany). The uterine cavity was distended and irrigated with a nonconductive, hypoosmolar solution of $2.7 \%$ sorbitol and $0.54 \%$ mannitol, instilled under manometric control by a hysteroscopic pump. The basic fluid setting was: intrauterine pressure $50 \mathrm{mmHg}$ (never exceeding $120 \mathrm{mmHg}$ during surgery), inflow $150 \mathrm{ml} / \mathrm{min}$, aspiration pressure 0.2 bar. The lowest pressure compatible with a clear vision was used during the procedure by "tailoring" opening and closure of inflow and outflow stopcocks.

The combined procedure consisted of a sequence of three different operating steps. At first, traditional slicing of the protruding intracavitary portion of the myoma is carried out by repeated and progressive passages of the semicircular monopolar cutting electrotome, with pure cutting current at $90 \mathrm{~W}$. This action stopped at the level of the plane of the endometrial surface so that the passage between the myoma and the adjacent myometrium was clearly identified (pseudocapule, i.e. cleavage plane). Secondly, cold enucleation of the intramural portion of the myoma was achieved by traction-and-leverage manoeuvres with rectangular and hooked non-electrical "cold" loops. Once the intracavitary portion was resected, the usual cutting loop was substituted on the same resectoscope by a suitable blunt dissection cold loop. By gentle traction on the myoma, the pseudocapsule was clearly identified and the cold loops were then inserted in this avascular space between the whitish and compact surface of the myoma and the pinkish and smooth adjacent myometrium. These loops were then progressively used in a mechanical way by hooking and lacerating the connective bridges joining the myoma to normal tissue (see video). Finally, the removal of the enucleated intramural portion was completed by progressive slicing, being now completely dislocated and therefore safely treatable as a lesion with a total intracavitary development and/or by using the rake cold loop (Mazzon Non-Electrical Loops, Karl Storz GmbH \& Co., Germany) (Fig. 1). All resected specimens were sent for histologic analysis.

Operating time was considered as the time between initial insertion and final removal of the resectoscope. Fluid deficit was calculated as the difference between the total amount of instilled solution into the uterus and the amount of fluid recovered from the suction bottle and the plastic draping that funnelled escaping fluid through the cervix into a calibrated pouch. According to the AAGL guidelines [16], a strict fluid monitoring was assessed every 10 $15 \mathrm{~min}$. In cases exceeding $60 \mathrm{~min}$ of intervention and/or a fluid deficit $>1,000 \mathrm{ml}$, intra-operative sonography was used to decide if the remaining part of the lesion might be removed in few additional minutes or if stopping the procedure. 
Fig. 1 a Myoma enucleation by pointed hooked knife-shaped loop (arrow), used to hook and lacerate the connective bridges which join the myoma to the adjacent myometrium. b Myoma enucleation by rectangular loop (arrow), used to identify the cleavage plane between the myoma and myometrium. c Myoma removal by rake loop (arrow), used to remove the residual deep portion of the myoma once enucleated. d Residual myometrial fovea after myoma removal by non-electrical loops
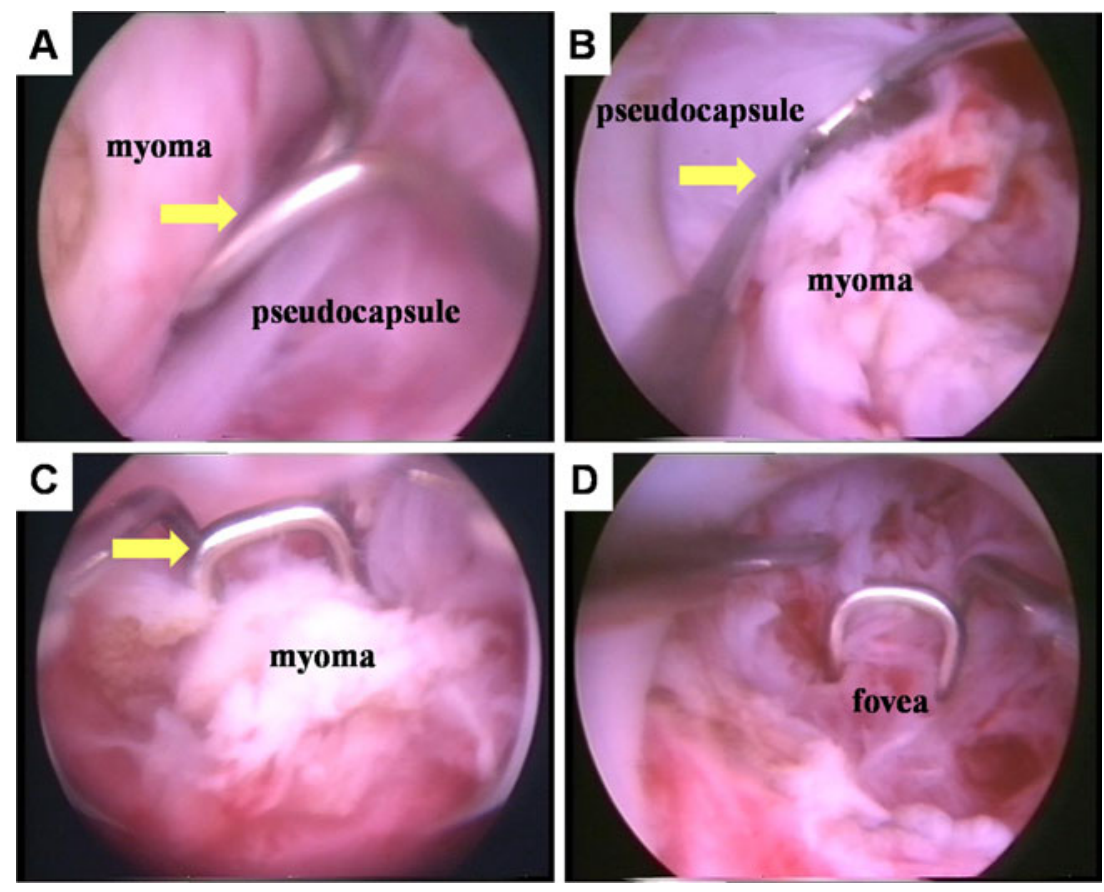

A short-term preoperative antimicrobial prophylaxis with cephalosporins was administered to all patients (cefazolin $1 \mathrm{~g}$ i.v.). All the surgical procedures were performed by one senior hysteroscopist (F.P.G.L.) in a day-surgery regimen. Within 2 months after the procedure, a SCSH was scheduled to evaluate disease persistence and to exclude uterine cavity lesions.

Intra-operative surgical outcomes were considered as follows: operating time, fluid deficit, surgical complications and complete resection at first procedure. Fluid deficit $>1,500 \mathrm{ml}$, perioperative haemorrhage and uterine perforation were considered as complications. Intrauterine lesions (synechiae) at follow-up and surgical and/or medical treatment for persisting uterine bleeding were considered as unfavourable long-term outcomes.

A telephone or direct interview follow-up after 6 and every 12 months was scheduled to assess menstrual pattern, pregnancy occurrence and outcome. All patients had at least a 1-year follow-up.

Analysis Demographic and baseline characteristics were analyzed by parametric and nonparametric descriptive statistics where appropriate. Continuous and dichotomous variables before and after surgery were compared using $t$ test, nonparametric test and the Fisher's exact test, respectively.

The 75th percentile of mean myoma diameters was used to define the categorical variable small/large myoma. A multivariate logistic analysis was performed to identify variables independently and significantly associated to surgical outcomes. Similarly, a multiple regression analysis was performed using myoma mean diameter and submucous myoma grading as continuous variables. Probability values less than $5 \%$ were considered significant. The time to event (abnormal uterine bleeding) analysis for all the patients was performed by the Kaplan-Meyer curve.

\section{Findings}

One hundred fifty-nine patients were recruited. Of these, 139 complained abnormal uterine bleeding, 6 were infertile and 14 had both symptoms. Median age (interquartile range) was 44 years (IR 35-48). Mean body mass index $( \pm \mathrm{SD})$ was $24 \pm 5 \mathrm{~kg} / \mathrm{m}^{2}$. Mean parity $( \pm \mathrm{SD})$ was $1 \pm 1$. The median mean myoma diameter was $2.1 \mathrm{~cm}$ (IR 1.6-3.0). Seventy-seven patients had additional intramural and/or subserous myomas and/or adenomyosis. Eighty-one patients $(51 \%)$ received preoperative medical treatment with GnRH analogue.

One hundred sixty-nine resectoscopic procedures were performed. Overall, 196 G1-G2 myomas were removed. In the 20 patients with multiple submucous myomas, the worst grading degree was considered for data analysis. Seventythree myomas were classified as G1 (46\%) and 86 as G2 (54\%). No women required perioperative blood transfusion. The histological report confirmed the diagnosis of leiomyoma in the vast majority of patients; only three cases of adenomyoma were reported. In 20 patients (13\%), concomitant simple endometrial hyperplasia was reported.

The multivariate logistic regression analysis of surgical results defined as perioperative outcomes (operating time 
$>50 \mathrm{~min}-75$ th percentile, fluid deficit $>500 \mathrm{ml}-75$ th percentile, incomplete resection, complications such as haemorrhage and uterine perforations), and as long-term outcomes (additional surgery and/or uterine cavity lesions), showed that myoma mean diameter $\geq 3 \mathrm{~cm}$ ( 75 th percentile) was the only significant independent variable associated to abnormal perioperative outcomes (Table 1). None of the considered variables were significantly associated with abnormal long-term surgical outcome. The mean $( \pm \mathrm{SD})$ myoma diameter of patients with perioperative unfavorable outcome (at least one abnormal outcome parameter) was $3.3 \mathrm{~cm}( \pm 1.0 \mathrm{~cm})$, significantly different to the $2.2 \mathrm{~cm}$ $( \pm 0.9 \mathrm{~cm})$ myoma mean diameter of patients with favourable outcome $(p \leq 0.001)$.

In order to strengthen the above results, a multiple regression analysis was performed. Myoma mean diameter and submucous myoma grading were considered as continuous variables in relation to both operating time and fluid deficit. By a $1-\mathrm{cm}$ increase of the myoma mean diameter, a significant mean operating time increase of $9.6 \mathrm{~min}$ was observed ( $p \leq 0.0001)$; conversely, a submucous myoma G2 required a not significant operative time increase of $4.4 \mathrm{~min}$ compared to a $\mathrm{G} 1(p=0.25)$. Similarly, by a $1-\mathrm{cm}$ increase of the myoma mean diameter, a significant mean fluid deficit increase of $190 \mathrm{ml}$ was observed $(p<0.001)$; conversely, a G2 required a not significant fluid deficit increase of $100 \mathrm{ml}$ compared to a $\mathrm{G} 1(p=0.06)$.

Table 2 reports surgical outcome of the whole study group and distinguished in details according to myoma grading and mean diameter. The prevalence of myomas $\geq 3 \mathrm{~cm}$ (75th percentile) was not significantly different between G1 and G2 myomas ( $19 \%$ vs. $27 \%$; $p=$ n.s.). One case of uterine perforation occurred by the non-electrical "cold" loop. In the absence of unpredictable thermal damage, this lesion was conservatively managed by transvaginal sonographic monitoring, sulprostone i.v. and prolonged antibiotic therapy.

We compared the first 50 consecutive procedures (learning curve period) with the remaining 119. Perioperative results in the learning period and at steady state were not significantly different both for G1 and G2 myomas and for myomas smaller and larger than $3 \mathrm{~cm}$.

Ten patients $(6 \%)$ were lost at follow-up.

Irregular bleeding persisted in $23 / 143$ patients $(16 \%)$, successfully controlled with medical therapy in 13 cases. Figure 2 shows the 7 years (75th percentile of follow-up) of cumulative proportion of women with recurrence of abnormal uterine bleeding. No significant differences were found in the surgery-free survival curve considering grading groups (G1 vs. G2) or myoma mean diameter groups ( $<3$ vs. $\geq 3.0 \mathrm{~cm})$.

At a median follow-up period of 63 months (IR 36-85), five patients required myomectomy and five hysterectomy for persisting symptoms. Intrauterine adhesions occurred in four of 156 patients (2.6\%), which were successfully removed by office hysteroscopy. One patient showed at SCSH a myometrial defect due to electrical damage.

Nine of the 20 infertile patients conceived. Three patients delivered vaginally at term; three patients delivered by caesarean section for breech presentation, foetal macrosomia and foetal distress during labour. One twin pregnancy and one singleton complicated by pre-eclampsia were delivered preterm by caesarean section. One patient had a first trimester miscarriage.

\section{Conclusions}

In this prospective study of 159 premenopausal patients with G1 and G2 submucous myomas, we report the combined use of electrical (slicing) and non-electrical loops (enucleation) to respect myometrial fibres functional anatomy and to reduce intravasation and bleeding. We obtained excellent surgical results with a high rate of single step complete resection (88\%) with minimal myometrial thermal injury with a negligible number of synechiae $(2.6 \%)$ and myometrial lesions $(0.6 \%)$. This compares well to the best

Table 1 Multivariate logistic regression analysis of perioperative and long-term surgical outcomes

\begin{tabular}{|c|c|c|c|c|c|c|}
\hline & \multicolumn{3}{|c|}{ Perioperative surgical outcomes (\#159) } & \multicolumn{3}{|c|}{ Long-term surgical outcomes (\#153) } \\
\hline & Odds ratio & $95 \% \mathrm{CI}$ & $p>\mathrm{Chi}^{2}$ & Odds ratio & $95 \% \mathrm{CI}$ & $p>\mathrm{Chi}^{2}$ \\
\hline Age ( $>35$ years) & 1.03 & $0.98-1.08$ & 0.29 & 0.99 & $0.91-1.08$ & 0.83 \\
\hline Myoma pre-surgical mean diameter $(\geq 3.0 \mathrm{~cm})$ & 8.2 & $1.13-21.0$ & 0.001 & 1.37 & $0.23-8.24$ & 0.73 \\
\hline Grading (G2) & 1.05 & $0.48-2.32$ & 0.90 & 0.61 & $0.16-2.32$ & 0.47 \\
\hline Number of submucous myomas $(>\# 1)$ & 2.4 & $0.86-6.85$ & 0.09 & 3.98 & $0.79-20.0$ & 0.09 \\
\hline Preoperative GnRH analogue (yes) & 1.08 & $0.45-2.55$ & 0.87 & 0.72 & $0.14-3.68$ & 0.69 \\
\hline Coexisting uterine lesions (yes) ${ }^{\mathrm{a}}$ & 0.96 & $0.46-2.0$ & 0.90 & 0.32 & $0.08-1.3$ & 0.11 \\
\hline
\end{tabular}

GnRH gonadotropin-releasing hormone

${ }^{\text {a }}$ Intramural myomas, adenomyosis and endometrial hyperplasia 
Table 2 Surgical outcomes of the whole study group (159 patients) and related to submucous myomas grading $(\mathrm{G})$ and to myoma mean diameter $(d)$

\begin{tabular}{|c|c|c|c|c|c|c|c|}
\hline & Study group & G1 & $\mathrm{G} 2$ & $p$ & $d<3.0 \mathrm{~cm}$ & $d \geq 3.0 \mathrm{~cm}$ & $p$ \\
\hline Patients, no. & 159 & 73 & 86 & - & 119 & 40 & - \\
\hline Procedures, no. & 169 & 76 & 93 & - & 123 & 46 & - \\
\hline Operating time (min) (median-IR) & $35(20-50)$ & $30(20-50)$ & $45(25-50)$ & 0.19 & $30(20-50)$ & $50(37-85)$ & 0.0001 \\
\hline Fluid deficit (ml) (median-IR) & $150(90-500)$ & $120(70-400)$ & $200(100-570)$ & 0.06 & $100(80-300)$ & $400(225-700)$ & 0.0001 \\
\hline Complications, no. $(\%)$ & $10(6)$ & $3(4)^{\mathrm{a}}$ & $7(8)^{\mathrm{b}}$ & 0.3 & $4(3)^{\mathrm{c}}$ & $6(15)^{\mathrm{d}}$ & 0.008 \\
\hline Complete resection at first procedure, no. (\%) & $140(88)$ & $67(92)$ & $73(85)$ & 0.18 & $116(96)$ & $24(60)$ & 0.0001 \\
\hline
\end{tabular}

IR interquartile range, $G 1$ grade $1, G 2$ grade 2

${ }^{a}$ Two fluid deficit $>1,500 \mathrm{ml}$, one perioperative haemorrhage

${ }^{\mathrm{b}}$ Three perioperative haemorrhage, one uterine perforation, three fluid deficit $>1,500 \mathrm{ml}$

${ }^{\mathrm{c}}$ Two fluid deficit $>1,500 \mathrm{ml}$, one uterine perforation, one perioperative haemorrhage

${ }^{\mathrm{d}}$ Three perioperative haemorrhage, three fluid deficit $>1,500 \mathrm{ml}$

reported results (1-12\%) and to unpublished data by Mazzon $[3,11,17]$.

In particular, the complete removal for G1 and G2 submucous myomas was $92 \%$ and $85 \%$, respectively, not significantly different. This result was in line with the best results of previous studies reporting a complete removal by traditional monopolar slicing of $73-93 \%$ for G1 and $50-85 \%$ for G2, respectively [4, 6, 7, 18-21].

By means of cold loops traction-and-leverage technique itself, combined with a basic low intrauterine pressure

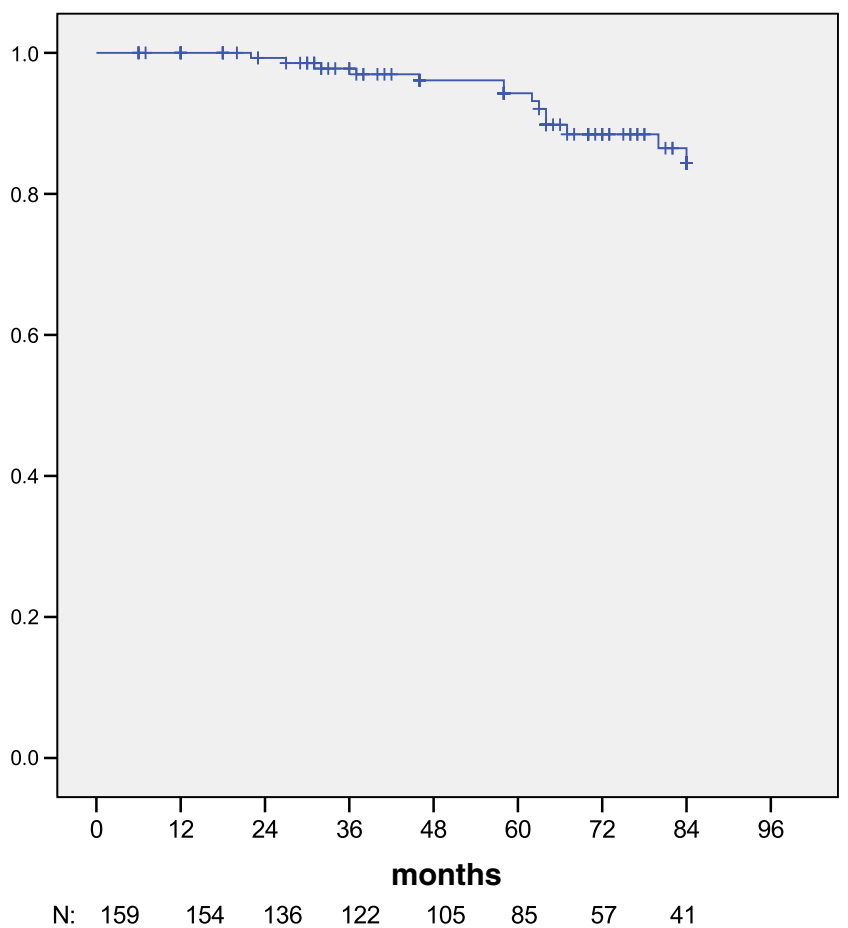

Fig. 2 Kaplan-Mayer curve of overall surgery-free survival. The percentage of patients free of surgery at 3 years was $97 \%(95 \% \mathrm{CI}$, 94-99.9), 94\% (95\% CI, 90-98) at 5 years and 87\% (95\% CI, 83-91) at 7 years setting $(50 \mathrm{mmHg})$, complete resections were more easily achieved also in large myomas, with a limited number of complications. In fact, the maintenance of the integrity of myometrial fibres, particularly the function of archimetrial layer [22], and of myoma pseudocapsule permitted to avoid deep and larger myometrial vessels damage and to obtain adequate haemostatic myometrial contractions. This reduced the risk of fluid overload, achieving a median fluid deficit significantly lower than in previous studies $[5,7]$ and similar to those reported more recently [21] which might have adopted similar low intrauterine fluid pressure.

The need of repetitive changing of electrical and nonelectrical "cold" loops during the same procedure might appear to be time-consuming. However, as well as in Formula 1 races, where the changing of tires at pit stop improves the car's performance, by this technique, the complete dislocation of the intramural portion of the myoma into the uterine cavity leads to a faster and safer complete removal.

To our surprise, myoma grading was not significantly associated with any of the adverse perioperative and longterm outcome. Beyond fluid deficit, which was nearly similar in G1 and G2, all other surgical outcomes were not affected by grading in this cohort in which myomas were enucleated by cold loops and not by electrosurgical slicing. The 75th percentile of myoma mean diameter of this cohort was $3 \mathrm{~cm}$. This occurred to be equal to the dimension cutoff value adopted by previous authors to differentiate small and large myomas $[6,21,23]$. This myoma mean diameter proved to be the only independent significant determinant correlated to perioperative surgical outcomes (operating time $>50 \mathrm{~min}$, fluid deficit $\geq 500 \mathrm{ml}$, surgical complications and incomplete resection at first procedure). These relevant data were strictly confirmed by the multiple regression analysis. Long-term outcomes (persisting symptoms and additional surgery for persisting symptoms) were not significantly different neither for the severity of grading 
nor for the myoma mean diameter and were comparable to those previously reported with a 7-year surgery-free rate of $87 \%[18,24]$.

Operation time, fluid decifit and complete resection at first procedure were highly significantly correlated to myoma dimension $>3 \mathrm{~cm}$. The importance of myomas size underlines the debatable role of pre-surgical medical treatment with the aim to reduce large submucous myomas $[25,26]$. GnRH analogues are significantly more efficacious on submucous when compared to the effect on intramural or subserosal myomas [27]. However, its use in this series was not significant (Table 1), probably due to the higher technical difficulties arising in the removal of the myoma from the pseudocapsule.

Perioperative complications (fluid deficit $>1,500 \mathrm{ml}$, uterine perforation and perioperative haemorrhage) occurred in ten cases $(6 \%)$. These data were comparable to reported rates in series considering advanced hysteroscopic procedures, i.e. involving the myometrium $[3,19,20]$.

The only uterine perforation occurred in a case with a coexisting intramural myoma adjacent to the submucous which prevented myometrial progressive thickening during enucleation as observed at sonography by Yang [28] and Casadio [29]. This perforation occurred by using cold loops avoiding further thermal damage on surrounding organs.

The negligible number of synechiae $(2.6 \%)$ and myometrial lesions $(0.6 \%)$ are strictly correlated with the surgical technique, clearly demonstrating that no thermal damage by monopolar current into the myometrium is an important factor for the adhesion formation, significantly lower than the reported variable incidence of $7.5-45.5 \%$ $[11,17,30]$. These results, added to the high rate of $\mathrm{G} 2$ and $>3 \mathrm{~cm}$ submucous myomas one-step complete resection ( $85 \%$ and $60 \%$, respectively) and the low rate of intraoperative complications, clearly evidenced the proven practical advantages derived by the use of this combined electrical and non-electrical "cold" technique. Obstetric outcomes in infertile patients were satisfactory, with a pregnancy rate of $45 \%$, similar to previously published data $(36-45 \%)[31,32]$.

Surgeon skills might be a remarkable bias in this series, being all procedures performed by an experienced hysteroscopist [3, 13, 23]. However, the learning curve analysis by comparing the first 50 procedures with the following did not detect any significant worse outcomes in the learning phase.

Although the study group was limited for the evaluation of short- and long-term complications, the population was very well defined and the surgical conditions were also well standardized (one technique, one operator and one centre).

In conclusion, hysteroscopic myomectomy by cold loops revealed to be an effective (low complication rate and high single-step complete resection) and efficient (day-surgery procedure and low long-term failure rate) surgical approach to the removal of submucous myoma with intramural development. Furthermore, by using this technique, myoma mean diameter proved to be the only prognostic factor of perioperative outcomes.

Conflicts of interest The authors report no conflicts of interest. The authors alone are responsible for the content and writing of the paper.

\section{References}

1. Hidlebaugh DA (2001) Relative costs of gynecologic endoscopy vs traditional surgery for treatment of abnormal uterine bleeding. Am J Manag Care 7:SP31-SP37

2. Brumsted JR, Blackman JA, Badger GJ, Riddick DH (1996) Hysteroscopy versus hysterectomy for the treatment of abnormal uterine bleeding: a comparison of cost. Fertil Steril 65(2):310-316

3. Di Spiezio SA, Mazzon I, Bramante S, Bettocchi S, Bifulco G, Guida M et al (2008) Hysteroscopic myomectomy: a comprehensive review of surgical techniques. Hum Reprod Update 14 (2):101-119

4. Wamsteker K, Emanuel MH, de Kruif JH (1993) Transcervical hysteroscopic resection of submucous fibroids for abnormal uterine bleeding: results regarding the degree of intramural extension. Obstet Gynecol 82(5):736-740

5. Emanuel MH, Hart A, Wamsteker K, Lammes F (1997) An analysis of fluid loss during transcervical resection of submucous myomas. Fertil Steril 68(5):881-886

6. Hart R, Molnar BG, Magos A (1999) Long term follow up of hysteroscopic myomectomy assessed by survival analysis. Br J Obstet Gynaecol 106(7):700-705

7. Vercellini P, Zaina B, Yaylayan L, Pisacreta A, De Giorgi O, Crosignani PG (1999) Hysteroscopic myomectomy: long-term effects on menstrual pattern and fertility. Obstet Gynecol 94 (3):341-347

8. Darwish A, Zareh HZ (2002) Haemodynamic, biochemical and haematological changes during hysteroscopic myomectomy. Gynecol Endosc 11:349-355

9. Baggish MS, Tucker RD (1995) Tissue actions of bipolar scissors compared with monopolar devices. Fertil Steril 63(2):422-426

10. Munro MG, Fu YS (1995) Loop electrosurgical excision with a laparoscopic electrode and carbon dioxide laser vaporization: comparison of thermal injury characteristics in the rat uterine horn. Am J Obstet Gynecol 172(4 Pt 1):1257-1262

11. Yang JH, Chen MJ, Wu MY, Chao KH, Ho HN, Yang YS (2008) Office hysteroscopic early lysis of intrauterine adhesion after transcervical resection of multiple apposing submucous myomas. Fertil Steril 89(5):1254-1259

12. Mazzon I (1995) Nuova tecnica per la miomectomia isteroscopica: enucleazione con ansa fredda.In Testo-Atlante di Chirurgia Endoscopica Ginecologica. Edited by E Cittadini, A Perino, M Angiolillo, et al. Palermo, Italy, Cofese Ed.

13. Camanni M, Bonino L, Delpiano EM, Ferrero B, Migliaretti G, Deltetto F (2010) Hysteroscopic management of large symptomatic submucous uterine myomas. J Minim Invasive Gynecol 17 (1):59-65

14. Leone FP, Lanzani C, Ferrazzi E (2003) Use of strict sonohysterographic methods for preoperative assessment of submucous myomas. Fertil Steril 79(4):998-1002

15. Leone FP, Bignardi T, Marciante C, Ferrazzi E (2007) Sonohysterography in the preoperative grading of submucous fibroids: 
considerations on three-dimensional methodology. Ultrasound Obstet Gynecol 29:717-718

16. Loffer FD, Bradley LD, Brill AI, Brooks PG, Cooper JM (2000) Hysteroscopic fluid monitoring guidelines. J Am Assoc Gynecol Laparosc 7(1):167-168

17. Touboul C, Fernandez H, Deffieux X et al (2009) Uterine synechiae after bipolar hysteroscopic resection of submucosal myomas in patients with infertility. Fertil Steril 92:1690

18. Emanuel MH, Wamsteker K, Hart AA, Metz G, Lammes FB (1999) Long-term results of hysteroscopic myomectomy for abnormal uterine bleeding. Obstet Gynecol 93(5 Pt 1):743-748

19. Cravello L, Agostini A, Beerli M, Roger V, Bretelle F, Blanc B (2004) Results of hysteroscopic myomectomy. Gynecol Obstet Fertil 32(9):825-828

20. Polena V, Mergui JL, Perrot N, Poncelet C, Barranger E, Uzan S (2007) Long-term results of hysteroscopic myomectomy in 235 patients. Eur J Obstet Gynecol Reprod Biol 130(2):232-237

21. Lasmar RB, Xinmei Z, Indman PD, Celeste RK, Di Spiezio SA (2011) Feasibility of a new system of classification of submucous myomas: a multicenter study. Fertil Steril 95(6):20732077

22. de Ziegler D, Bulletti C, Fanchin R, Epiney M, Brioschi PA (2001) Contractility of the nonpregnant uterus: the follicular phase. Ann N Y Acad Sci 943:172-184

23. Donnez J, Polet R, Smets M, Bassil S, Nisolle M (1995) Hysteroscopic myomectomy. Curr Opin Obstet Gyencol 7:311316

24. Cravello L, Farnarier J, Roger V, D’Ercole C, Blanc B (1998) Hysteroscopic myomectomy. Functional results with an average follow-up of 6 years. J Gynecol Obstet Biol Reprod (Paris) 27 (6):593-597
25. Muzii L, Boni T, Bellati F, Marana R, Ruggiero A, Zullo MA et al (2010) GnRH analogue treatment before hysteroscopic resection of submucous myomas: a prospective, randomized, multicenter study. Fertil Steril 94(4):1496-1499

26. Mavrelos D, Ben-Nagi J, Davies A, Lee C, Salim R, Jurkovic D (2010) The value of pre-operative treatment with GnRH analogues in women with submucous fibroids: a double-blind, placebocontrolled randomized trial. Hum Reprod 25(9):2264-2269

27. Brosens J, Campo R, Gordts S, Brosens I (2003) Submucous and outer myometrium leiomyomas are two distinct clinical entities. Fertil Steril 79(6):1452-1454

28. Yang JH, Lin BL (2001) Changes in myometrial thickness during hysteroscopic resection of deeply invasive submucous myomas. J Am Assoc Gynecol Laparosc 8(4):501-505

29. Casadio P, Youssef AM, Spagnolo E, Rizzo MA, Talamo MR, De Angelis D et al (2011) Should the myometrial free margin still be considered a limiting factor for hysteroscopic resection of submucous fibroids? A possible answer to an old question. Fertil Steril 95(5):1764-1768

30. Taskin O, Sadik S, Onoglu A, Gokdeniz R, Erturan E, Burak F, Wheeler JM (2000) Role of endometrial suppression on the frequency of intrauterine adhesions after resectoscopic surgery. $\mathrm{J}$ Am Assoc Gynecol Laparosc 7(3):351-354

31. Somigliana E, Vercellini P, Daguati R, Pasin R, De Giorgi O, Crosignani PG (2007) Fibroids and female reproduction: a critical analysis of the evidence. Hum Reprod Update 13:465-476

32. Shokeir T, El-Shafei M, Yousef H, Allam AF, Sadek E (2010) Submucous myomas and their implications in the pregnancy rates of patients with otherwise unexplained primary infertility undergoing hysteroscopic myomectomy: a randomized matched control study. Fertil Steril 94(2):724-729 